\title{
Oral Coadministration of Fluconazole with Tramadol Markedly Increases Plasma and Urine Concentrations of Tramadol and the O-Desmethyltramadol Metabolite in Healthy Dogs
}

\author{
(1)Tania E. Perez Jimenez, Butch Kukanich, Hyun Joo, Katrina L. Mealey, Tamara L. Grubb, \\ Stephen A. Greene, and Michael H. Court \\ Program in Individualized Medicine, Pharmacogenomics Laboratory, Department of Veterinary Clinical Sciences, Washington State \\ University College of Veterinary Medicine, Pullman, Washington (T.E.P.J., K.L.M., T.L.G., S.A.G., M.H.C.); and Department of \\ Anatomy and Physiology, Institute of Computational Comparative Medicine, Kansas State University College of Veterinary \\ Medicine, Manhattan, Kansas (B.K., H.J.) \\ Received July 5, 2018; accepted October 24, 2018
}

\section{ABSTRACT}

Tramadol is used frequently in the management of mild to moderate pain conditions in dogs. This use is controversial because multiple reports in treated dogs demonstrate very low plasma concentrations of $O$-desmethyltramadol (M1), the active metabolite. The objective of this study was to identify a drug that could be coadministered with tramadol to increase plasma M1 concentrations, thereby enhancing analgesic efficacy. In vitro studies were initially conducted to identify a compound that inhibited tramadol metabolism to $\mathrm{N}$-desmethyltramadol (M2) and M1 metabolism to N,O-didesmethyltramadol (M5) without reducing tramadol metabolism to $M 1$. A randomized crossover drug-drug interaction study was then conducted by administering this inhibitor or placebo with tramadol to 12 dogs. Blood and urine samples were collected to measure tramadol, tramadol metabolites, and inhibitor concentrations. After screening 86 compounds, fluconazole was the only drug found to inhibit M2 and M5 formation potently without reducing M1 formation. Four hours after tramadol administration to fluconazole-treated dogs, there were marked statistically significant $(P<0.001$; Wilcoxon signed-rank test) increases in plasma tramadol (31-fold higher) and M1 (39-fold higher) concentrations when compared with placebo-treated dogs. Conversely, plasma M2 and M5 concentrations were significantly lower (11-fold and 3-fold, respectively; $P<0.01)$ in fluconazole-treated dogs. Metabolite concentrations in urine followed a similar pattern. This is the first study to demonstrate a potentially beneficial drug-drug interaction in dogs through enhancing plasma tramadol and M1 concentrations. Future studies are needed to determine whether adding fluconazole can enhance the analgesic efficacy of tramadol in healthy dogs and clinical patients experiencing pain.
Introduction

Tramadol is frequently used in the management of mild to moderately painful conditions in dogs (Gaynor, 2008; Lamont, 2008). The acute analgesic properties of tramadol are thought to derive largely from effects on $\mu$-opioid receptors in the central nervous system (Raffa et al., 1992). Tramadol itself shows relatively weak affinity for opioid receptors in cell-based and animal studies. However, after administration, tramadol is metabolized by cytochrome P450 (P450) enzymes to $O$-desmethyltramadol (M1), which has about 200-times higher affinity for the $\mu$-opioid receptor than the parent compound (Raffa et al., 1992). Opioid receptor-independent effects of tramadol have also been identified, including inhibition of norepinephrine and serotonin reuptake

T.E.P.J. was supported in these studies by a Morris Animal Foundation Fellowship Training Grant [D16CA-401], and a Washington State University College of Veterinary Medicine Department of Veterinary Clinical Sciences graduate student grant. M.H.C. was supported by the National Institutes of Health [Grant GM102130] and the William R. Jones endowment to Washington State University College of Veterinary Medicine.

https://doi.org/10.1124/dmd.118.083444. transporters, which may contribute to analgesic effects, especially with chronic tramadol dosing (Raffa et al., 1992; Aronson, 1997).

Tramadol metabolism has been investigated extensively in humans, but only recently has it been studied in detail in veterinary medicine, particularly in dogs (Giorgi et al., 2009; Kukanich and Papich, 2011; Perez Jimenez et al., 2016). In addition to metabolism to M1, tramadol is metabolized extensively to $N$-desmethyltramadol (M2). Both M1 and $\mathrm{M} 2$ are also further metabolized to $\mathrm{N}, \mathrm{O}$-didesmethyltramadol (M5), which is the major metabolite found in the plasma and urine of dogs given tramadol (Kukanich and Papich, 2011). Tramadol, M1, M2, and M5 can also be further metabolized by conjugation, including both glucuronidation and sulfation (Grond et al., 1999).

One consistent finding of these pharmacokinetic studies is the relatively low circulating concentrations of M1 in dogs when compared with other species, including humans (Scott and Perry, 2000) and cats (Pypendop et al., 2009), even when adjusted for differences in tramadol dose. Such low concentrations of M1 in dogs may have clinical consequences in that multiple studies have failed to demonstrate significant analgesic effects, at least when compared with other commonly used analgesic drugs (Davila et al., 2013; Delgado et al.,

ABBREVIATIONS: DLM, dog liver microsome; GMR, geometric mean ratio; HPLC, high-performance liquid chromatography; M1, Odesmethyltramadol; M2, N-desmethyltramadol; M5, N,O-didesmethyltramadol; MS, mass spectrometry; m/z, mass-to-charge ratio; P450, cytochrome P450. 
2014; Kögel et al., 2014). In contrast, studies of tramadol in cats have consistently demonstrated analgesic efficacy (Pypendop et al., 2009; Evangelista et al., 2014), which is accompanied by much higher plasma M1 concentrations (Pypendop et al., 2009; Cagnardi et al., 2011). Consequently, increasing plasma M1 concentrations such as through selective inhibition of tramadol and M1 metabolic pathways is a plausible strategy that could lead to enhanced analgesic effects of tramadol in dogs.

In previous studies, we demonstrated that tramadol is metabolized by dog liver microsomes (DLMs) to M1 mainly by CYP2D15 and to M2 by CYP2B11 and CYP3A12, which are species orthologs of CYP2D6, CYP2B6, and CYP3A4, respectively, that perform the same function in humans (Subrahmanyam et al., 2001; Perez Jimenez et al., 2016). We also went on to show that CYP2C21 is mainly responsible for the formation of M5 from M1 and that CYP2D15 is exclusively responsible for formation of M5 from M2 (Perez Jimenez et al., 2018). P450 phenotyping of these secondary pathways has not yet been reported for human enzymes, as far as we are aware.

The purpose of this study was to identify a drug that could be coadministered with tramadol to increase plasma M1 concentrations, thereby enhancing analgesic effectiveness. We used a two-step approach. First, we conducted an in vitro screen using DLMs to identify a drug that selectively inhibits M2 formation from tramadol and M5 formation from M1, without inhibiting M1 formation from tramadol. We then conducted a proof-of-principle drug-drug interaction study in healthy dogs to test the hypothesis that the identified drug would increase plasma and urine concentrations of tramadol and M1, while decreasing concentrations of M2 and M5.

\section{Materials and Methods}

Reagents. $\mathrm{NADP}^{+}$, isocitrate dehydrogenase, DL-isocitrate, and fluconazole were obtained from Sigma-Aldrich (St. Louis, MO). (+)-Tramadol hydrochloride, (-)-tramadol, $O$-desmethyltramadol hydrochloride, $O$-desmethyltramadol-D ${ }_{6}$, $\mathrm{N}$-desmethyltramadol, $\mathrm{N}$-desmethyltramadol- $\mathrm{D}_{3}$ hydrochloride, and $\mathrm{N}, \mathrm{O}$-didesmethyl tramadol-d3 hydrochloride were purchased from Toronto Research Chemicals Inc. (Toronto, Ontario, Canada). Tramadol $-{ }^{13} \mathrm{C}, \mathrm{D}_{3} \mathrm{HCl}$ was obtained from Cerilliant (Round Rock, TX). Racemic ( \pm )-tramadol was made by combining equal amounts of $(+)$-tramadol and $(-)$-tramadol.

Liver microsomes were prepared as previously described (Court et al., 1997) from a bank of frozen dog livers maintained at Washington State University. Livers were from 59 untreated adult dogs including 25 beagles (six females, 19 males), five greyhounds (all males), 12 mixed breed dogs (six females and six males), four Chihuahuas (three males and one female), 12 hounds (all females), and one Labrador retriever (male). All dogs were healthy and were euthanized for reasons unrelated to this study. The collection of the dog livers was approved by the Institutional Animal Care and Use Committee Washington State University. The microsomal protein content of the DLMs used in this study was measured using the bicinchoninic acid assay (Thermo Scientific Pierce, Rockford, IL).

Assay to Screen for Inhibitors of M1 and/or M2 Formation from Tramadol. Eighty-five compounds (listed in Table 1) were screened as potential inhibitors of M1 and M2 formation from tramadol in pooled DLMs. These compounds were obtained as pure chemicals from Sigma-Aldrich, or from the following sources. Acepromazine maleate (PromAce) and meloxicam (Metacam) were obtained from Boehringer Ingelheim Vetmedica Inc. (St. Joseph, MO). Atipamezole (antisedan) and dexmedetomidine $\mathrm{HCl}$ (Dexdomitor) were obtained from Orion Pharma (Espoo, Finland). Bupivacaine HCL (Sensorcaine) was obtained from APP Pharmaceuticals LLC (Lake Zurich, IL). Propofol (Propoflo28) was obtained from Abbott Laboratories (North Chicago, IL). Lidocaine $\mathrm{HCl}$ and fentanyl citrate were obtained from Hospira, Inc. (Lake Forest, IL). Carprofen (Rimadyl), maropitant citrate (Cerenia), and ketamine $\mathrm{HCl}$ (Ketaset) were obtained from Zoetis Inc. (Kalamazoo, MI). Hydromorphone $\mathrm{HCl}$ was obtained from West-Ward (Eatontown, NJ). Midazolam was obtained from Akorn Inc. (Lake Forest, IL). Alfaxalone (Alfaxan) was obtained from Jurox Pty Limited (Rutherford, NSW, Australia). All compounds were dissolved in methanol at two concentrations ( 1 and $100 \mu \mathrm{M}$ ), added to $2 \mathrm{ml}$ polypropylene Eppendorf incubation tubes, and dried in a centrifugal vacuum. NADPH cofactor mix and $20 \mu \mathrm{g}$ of DLMs were added to the tubes and preincubated at $37^{\circ} \mathrm{C}$ for 15 minutes. Tramadol ( $5 \mu \mathrm{M}$ final concentration) was then added and incubated for a further 10 minutes. The reaction was stopped by adding internal standard and the metabolites formed were measured by high-performance liquid chromatography (HPLC) mass spectrometry (MS) as described previously (Perez Jimenez et al., 2016).

Determination of Potency of Inhibition by Fluconazole. The potency [measured as half-maximal inhibitory concentration $\left(\mathrm{IC}_{50}\right)$ values] of fluconazole as an inhibitor of M1 and M2 formation from tramadol and M5 formation from M1 and M2 was determined at multiple concentrations in pooled DLMs. Fluconazole was dissolved in methanol at $0.001,0.01,0.1,1,10,100$, and $1000 \mu \mathrm{M}$ concentrations, added to incubation tubes, and dried in a centrifugal vacuum. NADPH cofactor mix and enzyme ( $20 \mu \mathrm{g}$ of DLMs for tramadol metabolism to M1 and M2, $10 \mu \mathrm{g}$ of DLMs for M1 metabolism to M5, and $5 \mu \mathrm{g}$ of DLMs for M2 metabolism to M5 formation) were added to the tube and preincubated at $37^{\circ} \mathrm{C}$ for 15 minutes. Substrates including tramadol $(5 \mu \mathrm{M}), \mathrm{M} 1$ $(10 \mu \mathrm{M})$, and $\mathrm{M} 2(0.5 \mu \mathrm{M})$ were then added and incubated for a further 10, 20, and 5 minutes, respectively. The reaction was stopped by adding internal standard and the metabolites formed were measured by HPLC-MS as described in detail previously (Perez Jimenez et al., 2016, 2018). Samples were prepared in triplicate. The M1, M2, and M5 formation rates were averaged, and then expressed as a percentage of control incubations that lacked inhibitor. The $\mathrm{IC}_{50}$ values were determined using nonlinear regression with a four-parameter logistic curve (Sigmaplot version 13 software; Systat, San Jose, CA).

An inhibition constant $\left(K_{\mathrm{i}}\right)$ was also determined for the inhibition of M2 formation from tramadol in DLMs by using varying tramadol concentrations of tramadol $(0,5,10,20,50,100,200$, and $500 \mu \mathrm{M})$ and fluconazole concentrations $(0,0.1,1,10$, and $100 \mu \mathrm{M})$. Incubations and HPLC-MS analysis were performed as previously described. Nonlinear regression analysis (Sigmaplot version 13 software; Systat) was used to derive fitted parameter estimates, including $K_{\mathrm{i}}$, $K_{\mathrm{m}}$, and $V_{\max }$, employing the competitive, noncompetitive, uncompetitive, and mixed competitive-noncompetitive enzyme inhibition models and the approach described previously (Venkatakrishnan et al., 2001).

In Vivo Drug-Drug Interaction Study. We conducted a two-way randomized crossover drug-drug interaction trial with a blinded observer. Based on published data (Kukanich and Papich, 2011) the mean and S.D. values of the plasma M1 concentrations were 6.3 and $2.9 \mathrm{ng} / \mathrm{ml}$, respectively. We proposed to detect a minimum $50 \%$ change in M1 concentration, with a 0.85 power, at a $P$ value of less than 0.05 using a paired $t$ test. Although sample size analysis indicated that we would need to study at least $10 \mathrm{dogs}$, we elected to study $12 \mathrm{dogs}$ to allow for up to two potential subject dropouts before the end of the study. This study was reviewed and approved by the Institutional Animal Care and Use Committee at Washington State University. All owners gave written informed consent for their dog to participate.

Study Subjects. Twelve healthy, owned dogs were recruited from among the staff, faculty, and students at the College of Veterinary Medicine at Washington State University (Pullman, WA). Health was determined by physical examination, history, complete blood count, and serum biochemistry profile, performed at the Veterinary Teaching Hospital, Washington State University. Dogs were between 2.5 and 7 years old (average 4 years old), included six males and six females, weighed between 20 and $35 \mathrm{~kg}$ (average $26 \mathrm{~kg}$ ), and had not been treated with any other medications within 2 weeks prior to or at the time of the study. Various dog breeds were represented, including Labrador retriever mix (3), German shepherd (1), flat-coated retriever (1), Australian shepherd mix (1), pit bull mix (1), Australian cattle dog/heeler mix (1), boxer mix (1), golden retriever mix (1), German shepherd mix (1), and wire-haired pointing griffon (1). All dogs were fasted by the owners on the morning of the study and were housed in kennels in the Veterinary Teaching Hospital at Washington State University. Blood sampling was performed in a room designated for the study inside the hospital.

Study Design. Pill pockets (Canine Greenies; The Nutro Company, Franklin, TN) were filled by the Veterinary Teaching Hospital pharmacist with either placebo (empty gelatin capsules) or fluconazole (4 mg/kg dose; WellSpring Pharmaceutical, Ontario, Canada). These were labeled as treatments A and B, respectively, and this code was maintained by the pharmacist until the completion of the study. The filled pill pockets were given to the owners to be administered at approximately 24 hours (the prior morning) and 12 hours (the prior night) before 
TABLE 1

Results of an in vitro screening assay to identify compounds that selectively inhibit M2 formation (but not M1 formation) from tramadol by dog liver microsomes

Shown are the formation rates of M1 and M2 from tramadol in pooled DLMs incubated with each of 85 different compounds at 1 and $100 \mu M$ inhibitor concentrations. Results are presented as the percentage of control (no inhibitor added) activity and are the average of triplicate determinations conducted in a single experiment.

\begin{tabular}{|c|c|c|c|c|}
\hline \multirow{2}{*}{ Inhibitor } & \multicolumn{2}{|c|}{ M1 Formation (\% Control) } & \multicolumn{2}{|c|}{ M2 Formation (\% Control) } \\
\hline & $1 \mu \mathrm{M}$ Inhibitor & $100 \mu \mathrm{M}$ Inhibitor & $1 \mu \mathrm{M}$ Inhibitor & $100 \mu \mathrm{M}$ Inhibitor \\
\hline Acepromazine & 92 & 20 & 94 & 84 \\
\hline Acetaminophen & 95 & 92 & 93 & 95 \\
\hline Alfaxalone & 93 & 51 & 90 & 68 \\
\hline Amytriptylline & 95 & 0.9 & 148 & 25 \\
\hline Artemisinin & 95 & 66 & 73 & 7 \\
\hline Acetylsalicylic acid & 93 & 94 & 91 & 96 \\
\hline Atipamezole & 18 & 0.1 & 6 & 5 \\
\hline Bupivacaine & 32 & 1 & 91 & 23 \\
\hline Buprenorphine & 84 & 4 & 97 & 43 \\
\hline Bupropion & 94 & 16 & 93 & 47 \\
\hline Carbamazepine & 95 & 54 & 91 & 52 \\
\hline Carprofen & 96 & 61 & 95 & 46 \\
\hline Chloramphenicol & 100 & 44 & 95 & 13 \\
\hline Cimetidine & 99 & 37 & 97 & 65 \\
\hline Ciprofloxacin & 95 & 77 & 91 & 92 \\
\hline Citalopram & 6 & 0.3 & 92 & 13 \\
\hline Clarithromycin & 99 & 95 & 104 & 98 \\
\hline Clopidrogel & 95 & 28 & 62 & 8 \\
\hline Codeine & 100 & 89 & 99 & 78 \\
\hline Curcumin & 96 & 48 & 94 & 59 \\
\hline Cyclophosphamide & 90 & 89 & 85 & 76 \\
\hline Deracoxib & 78 & 9 & 77 & 20 \\
\hline Desipramine & 37 & 1 & 49 & 6 \\
\hline Dexamethasone & 91 & 67 & 92 & 81 \\
\hline Dexmedetomidine & 99 & 86 & 90 & 8 \\
\hline Dextrometorphan & 65 & 1 & 90 & 30 \\
\hline Diazepam & 93 & 32 & 81 & 10 \\
\hline Diclofenac & 101 & 136 & 101 & 71 \\
\hline Diethyldithiocarbamate & 93 & 80 & 95 & 65 \\
\hline Dopamine & 95 & 46 & 97 & 89 \\
\hline Doxepin & 82 & 3 & 90 & 36 \\
\hline Doxycycline & 97 & 73 & 98 & 88 \\
\hline Diphenhydramine & 87 & 5 & 90 & 37 \\
\hline Efavirenz & 122 & 48 & 52 & 10 \\
\hline Erythromycin & 94 & 89 & 100 & 92 \\
\hline Fenoprofen & 93 & 61 & 90 & 71 \\
\hline Fentanyl & 82 & 12 & 88 & 67 \\
\hline Firocoxib & 90 & 44 & 97 & 78 \\
\hline Fluconazole & 97 & 14 & 10 & 3 \\
\hline Flurbiprofen & 94 & 145 & 102 & 99 \\
\hline Fluoxetine & 62 & 2 & 81 & 12 \\
\hline Furosemide & 90 & 86 & 96 & 85 \\
\hline Gabapentin & 102 & 88 & 101 & 88 \\
\hline Hydromorphone & 89 & 55 & 88 & 78 \\
\hline Ibuprofen & 95 & 81 & 89 & 89 \\
\hline Imipramine & 48 & 1 & 93 & 81 \\
\hline Itraconazole & 87 & 71 & 84 & 63 \\
\hline Ketamine & 94 & 45 & 62 & 14 \\
\hline Ketoconazole & 47 & 2 & 84 & 19 \\
\hline Ketoprofen & 94 & 83 & 92 & 60 \\
\hline Ketorolac & 94 & 89 & 90 & 84 \\
\hline Ketotifen & 63 & 2 & 85 & 25 \\
\hline Lamotrigine & 92 & 54 & 110 & 81 \\
\hline Levomedetomidine & 36 & 0.2 & 10 & 9 \\
\hline Lidocaine & 91 & 50 & 91 & 73 \\
\hline Lorazepam & 89 & 16 & 108 & 70 \\
\hline Maropitant & 7 & 1 & 82 & 63 \\
\hline Meloxicam & 93 & 83 & 90 & 80 \\
\hline Methadone & 67 & 2 & 87 & 21 \\
\hline Metoclopramide & 106 & 43 & 105 & 106 \\
\hline Metronidazole & 105 & 99 & 106 & 79 \\
\hline Midazolam & 91 & 5 & 69 & 24 \\
\hline Morphine & 95 & 85 & 96 & 91 \\
\hline Mycophenolic acid & 90 & 60 & 117 & 79 \\
\hline Naproxen & 90 & 65 & 114 & 99 \\
\hline Norfloxacin & 96 & 82 & 98 & 87 \\
\hline Ofloxacin & 94 & 82 & 94 & 88 \\
\hline Omeprazole & 105 & 28 & 112 & 57 \\
\hline Oxazepam & 92 & 39 & 139 & 78 \\
\hline
\end{tabular}


TABLE 1-Continued

\begin{tabular}{|c|c|c|c|c|}
\hline \multirow{2}{*}{ Inhibitor } & \multicolumn{2}{|c|}{ M1 Formation (\% Control) } & \multicolumn{2}{|c|}{ M2 Formation (\% Control) } \\
\hline & $1 \mu \mathrm{M}$ Inhibitor & $100 \mu \mathrm{M}$ Inhibitor & $1 \mu \mathrm{M}$ Inhibitor & $100 \mu \mathrm{M}$ Inhibitor \\
\hline Paroxetine & 27 & 1 & 20 & 11 \\
\hline Phenacetin & 85 & 49 & 124 & 79 \\
\hline Piperine & 84 & 70 & 126 & 76 \\
\hline Propofol & 93 & 72 & 91 & 22 \\
\hline Quinidine & 26 & 2 & 102 & 77 \\
\hline Quinine & 27 & 0.2 & 57 & 12 \\
\hline Selegiline & 87 & 50 & 92 & 20 \\
\hline Suprofen & 95 & 99 & 85 & 87 \\
\hline Terbinafine & 91 & 40 & 99 & 63 \\
\hline Thio-TEPA & 99 & 100 & 98 & 90 \\
\hline Trazadone & 103 & 31 & 110 & 54 \\
\hline Trifluoperazine & 34 & 1 & 88 & 25 \\
\hline Valproic Acid & 97 & 99 & 98 & 88 \\
\hline Vinblastine & 95 & 21 & 99 & 87 \\
\hline Xylazine & 93 & 17 & 97 & 41 \\
\hline Zomepirac & 96 & 93 & 95 & 77 \\
\hline
\end{tabular}

Values in bold indicate more than $50 \%$ inhibition.

the day of the testing. Randomization of the initial treatment that each dog received was achieved by drawing from a bag a piece of paper labeled with the treatment identification (A: placebo; B: fluconazole). Six dogs received treatment A first, while six dogs received treatment B first. On the morning of the day of testing the dogs were given a third pill pocket containing the assigned treatment (A or B), and 30 minutes later another pill pocket containing tramadol $(5 \mathrm{mg} / \mathrm{kg}$ dose; Amneal Pharmaceuticals, Hauppauge, NY) was administered. There was a minimum 2-week washout period between treatments to account for possible residual drug effect. The code identifying the treatments as either placebo or fluconazole was revealed by the pharmacist after completion of sample and data analysis.

Heparinized $5 \mathrm{ml}$ blood samples were collected aseptically from a peripheral vein immediately before tramadol administration (time 0 ) and 1 and 4 hours after tramadol administration. Urine samples were taken by free catch 4 hours after tramadol administration. Blood plasma was separated shortly after collection, and stored with the urine samples at $-80^{\circ} \mathrm{C}$ until assay for drug and metabolite concentrations by HPLC-MS.

Assays for Drug and Metabolite Concentrations in Plasma and Urine Samples. Plasma and urine samples were assayed for tramadol, M1, M2, and M5 concentrations with and without deconjugation. The deconjugation solution was sodium acetate buffer $(200 \mathrm{mM}, \mathrm{pH} 5.0)$, which contained $1666 \mathrm{U} / \mathrm{ml}$ of $\beta$-glucuronidase $(\beta$-glucuronidase type L-II from limpets, G8132; SigmaAldrich). For each sample, $150 \mu \mathrm{l}$ of the deconjugation solution was added to $50 \mu \mathrm{l}$ of plasma or urine and incubated for 3 hours at $65^{\circ} \mathrm{C}$. Untreated plasma and urine samples were mixed with the same buffer without $\beta$-glucuronidase added, and incubated similarly. An aliquot $(50 \mu \mathrm{l})$ of the incubation sample was then added to $100 \mu \mathrm{l}$ of $0.1 \%(\mathrm{v} / \mathrm{v})$ formic acid in water containing the internal standards (at $200 \mathrm{ng} / \mathrm{ml}$ each). Internal standards were tramadol- ${ }^{13} \mathrm{C}_{,} \mathrm{D}_{3}$ (for tramadol), M1-D (for M1 and M5), and M2-D (for M2). Phosphoric acid ( $150 \mu \mathrm{l}$ at $4 \% \mathrm{v} / \mathrm{v}$ in water) was then added and mixed. Elution plates (Oasis MCX $50 \mu$; Waters Corporation, Milford, MA) were first conditioned with $150 \mu \mathrm{l}$ of methanol, followed by $150 \mu 1$ deionized water. The samples were passed through the plate and washed with $150 \mu \mathrm{l}$ of $2 \%(\mathrm{v} / \mathrm{v})$ formic acid in deionized water followed by $150 \mu \mathrm{l}$ of methanol. The samples were then eluted with two consecutive $25 \mu \mathrm{l}$ volumes of $5 \%$ (v/v) ammonium hydroxide in methanol. The eluate was diluted with $100 \mu \mathrm{l}$ of deionized water prior to analysis by liquid chromatography-mass spectrometry.

Plasma sample preparation for measurement of fluconazole concentrations involved mixing $15 \mu \mathrm{l}$ of sample with $35 \mu \mathrm{l}$ untreated (blank) plasma and $100 \mu \mathrm{l}$ of acetonitrile with $1 \%$ formic acid containing the internal standard voriconazole at $1 \mu \mathrm{g} / \mathrm{ml}$. This was passed through a phospholipid removal plate (Ostro Passthrough Plates; Waters Corporation) and $2 \mu$ l of the eluate was injected into the liquid chromatography-mass spectrometry instrument.

Drug and metabolite concentrations were determined using a liquid chromatography instrument (Acquity UPLC H-Class UPLC; Waters Corporation) with a triple quadrupole mass spectrometry detector (TQD; Waters
Corporation). The mobile phase included: A, $0.1 \%$ formic acid in deionized water, and B, acetonitrile at a flow rate of $0.6 \mathrm{ml} / \mathrm{min}$. A gradient was used that began with $85 \% \mathrm{~A}$ to $5 \% \mathrm{~A}$ at 0.8 minutes, which was held until 1.3 minutes, followed by a return to $85 \% \mathrm{~A}$ at 1.31 minutes with a total run time of 2.2 minutes. The column (Acquity UPLC HSS T3 $1.8 \mu \mathrm{m}$; Waters Corporation) was heated to $40^{\circ} \mathrm{C}$. The mass-to-charge ratio $(\mathrm{m} / \mathrm{z})$ for the qualifying and quantifying positive ions $(\mathrm{m} / \mathrm{z}$ qualifying ion $\rightarrow$ quantifying ions) were as follows: fluconazole $=\mathrm{m} / \mathrm{z} 307.27 \rightarrow 169.06,220.14$; voriconazole $=m / z \quad 350.09 \rightarrow 126.97,281.06 ;$ tramadol $=m / z 264.20 \rightarrow$ 58.06; tramadol- ${ }^{13} \mathrm{C}_{1} \mathrm{D}_{3}=\mathrm{m} / \mathrm{z} 268.21 \rightarrow 58.05,91.96 ; \mathrm{M} 1=\mathrm{m} / \mathrm{z}, 250.12 \rightarrow$ $57.99 ; \mathrm{M} 1-\mathrm{D}_{6}=m / z 256.18 \rightarrow 45.65,63.98 ; \mathrm{M} 2=m / z 250.12 \rightarrow 44.04 ; \mathrm{M} 2-\mathrm{D}_{3}=$ $\mathrm{m} / \mathrm{z} 253.14 \rightarrow 47.04,216.88 ;$ and M5 $=\mathrm{m} / \mathrm{z} 236.10 \rightarrow 43.97,61.96$.

The accuracy and coefficient of variation (precision) of the assay were determined using quality control samples dissolved in canine plasma or urine prepared in replicates of three at multiple concentrations. The accuracy percentages for plasma samples (untreated and deconjugated) were 106\%, 98\%, $102 \%$, and $106 \%$ for tramadol, M1, M2, and M5, respectively, at 5, 50, and $500 \mathrm{ng} / \mathrm{ml}$ concentrations. The precision percentages of the assay were $5.6 \%$, $3.2 \%, 13 \%$, and $5.8 \%$ for tramadol, M1, M2, and M5, respectively, at 5, 50, and $500 \mathrm{ng} / \mathrm{ml}$ concentrations. The assay was linear over the quantified range of 1$1000 \mathrm{ng} / \mathrm{ml}$. The accuracy percentages of the assay for urine (untreated and deconjugated) were $102 \%, 95 \%, 102 \%$, and $103 \%$ for tramadol, M1, M2, and M5, respectively, at $4,40,400$, and $4000 \mathrm{ng} / \mathrm{ml}$ concentrations. The precision percentages of the assay were $1.2 \%, 2.3 \%, 3.3 \%$, and $7.6 \%$ for tramadol, M1, M2, and M5, respectively, at 4, 40, 400, and $4000 \mathrm{ng} / \mathrm{ml}$ concentrations. The assay was linear over the quantified range of $0.8-8000 \mathrm{ng} / \mathrm{ml}$. The accuracy of the plasma fluconazole assay was $98 \%$ and the precision was $5 \%$ at 400,2000 , and $8000 \mathrm{ng} / \mathrm{ml}$. The assay was linear over the quantified range of $400-10,000 \mathrm{ng} / \mathrm{ml}$.

Tramadol and metabolite conjugate concentrations (expressed as mass equivalents of the parent compound) were calculated by subtracting the untreated concentration from the $\beta$-glucuronidase-treated concentration for each plasma and urine sample.

Statistical Analyses. Statistical analyses were performed using Sigmaplot 13 software (Systat). Graphs were prepared using GraphPad Prism 7 (GraphPad Software Inc., La Jolla, CA). Data were summarized as the median and $25 \%-75 \%$ interquartile range. The geometric mean ratio (GMR) and the $90 \%$ confidence interval of the GMR were also calculated for plasma and urine drug metabolite concentrations obtained after fluconazole pretreatment divided by concentrations obtained after the placebo pretreatment (in the same animal). Plasma and urine concentrations that were less than the lower limit of quantitation were set to zero for descriptive statistics and were set to one-half of the lower limit of quantitation value to calculate the GMR. Paired $t$ tests or Wilcoxon signed-rank tests were used to compare pharmacokinetic data between fluconazole and placebo pretreatments. The Wilcoxon signed-rank test was used instead of the paired $t$ test if the data set failed the Shapiro-Wilk test for normality or the Brown-Forsythe test for equal variance. Data were rank transformed if the data set failed the Shapiro-Wilk test 
for normality or the Brown-Forsythe test for equal variance. A $P$ value of less than 0.05 was considered statistically significant.

\section{Results}

In Vitro Screen to Identify Selective Inhibitors of Tramadol Metabolic Pathways. An in vitro approach with pooled DLMs was used initially to identify a compound that could potently inhibit M2 formation from tramadol and M5 formation from M1 without affecting M1 formation from tramadol. Eighty-six compounds that could potentially be administered simultaneously with tramadol in the clinical setting, including antibiotics, sedatives, chemotherapeutics, anesthetics, analgesics, anticonvulsants, antidepressants, and antifungals (among others), were initially screened at two concentrations ( 1 and $100 \mu \mathrm{M})$ for their ability to inhibit M1 and M2 formation from tramadol in DLMs when compared with incubations without any inhibitor added (Table 1). Compounds were then categorized according to their inhibition potency (ability to decrease activity by more than $50 \%$ at 1 or $100 \mu \mathrm{M}$ inhibitor concentration) and by their selectivity (ability to inhibit by more than $50 \%$ M1 formation only, M2 formation only, or both M1 and M2 formation). As shown in Table 2, fluconazole was the only compound out of the 85 tested that decreased M2 formation from tramadol by more than $50 \%$ at the lowest inhibitor concentration tested $(1 \mu \mathrm{M})$ without affecting M1 formation (less than 50\% decrease) at the highest concentration tested $(100 \mu \mathrm{M})$.

These results for fluconazole were then corroborated by evaluating the rates of formation of M1 and M2 from tramadol in DLMs over a much wider range of fluconazole concentrations $(0.001-1000 \mu \mathrm{M})$ to estimate $\mathrm{IC}_{50}$ values. We also evaluated effects on M5 formation from both M1 and M2 using a similar approach to determine whether fluconazole would also selectively inhibit M1 N-demethylation. As shown in Fig. 1A, fluconazole potently and selectively inhibited the formation of M2 from tramadol $\left(\mathrm{IC}_{50}=0.40 \pm 0.04 \mu \mathrm{M}\right)$ and M5 formation from M1 $\left(\mathrm{IC}_{50}=0.24 \pm 0.02 \mu \mathrm{M}\right)$ when compared with the much lower potency of inhibiting M1 formation from tramadol $\left(\mathrm{IC}_{50}=102 \pm 34 \mu \mathrm{M}\right)$ and M5 formation from $\mathrm{M} 2\left(\mathrm{IC}_{50}=75 \mu \mathrm{M}\right)$.

Finally, the mechanism of inhibition of fluconazole and the inhibition constant $\left(K_{\mathrm{i}}\right)$ were determined for M2 formation from tramadol by evaluating multiple inhibitor and substrate concentrations and determining the inhibition model that best fit the data. As shown in Fig. 1B, a competitive inhibition model best described the data $\left(K_{\mathrm{m}}=73 \pm 8.8\right.$ $\mu \mathrm{M} ; V_{\max }=479 \pm 18 \mathrm{pmol} / \mathrm{min}$ per milligram protein, $K_{\mathrm{i}}=0.11 \pm 0.02$ $\mu \mathrm{M}$; adjusted $\left.R^{2}=0.9809 ; P<0.0001\right)$. Unfortunately, it was not possible to determine the $K_{\mathrm{i}}$ value and mechanism of inhibition by fluconazole of M5 formation from M1 because of the slow reaction velocity, which greatly limited assay precision and accuracy.

Fluconazole-Tramadol Interaction In Vivo Study. All 12 enrolled dogs completed the study. Only minor adverse events, including mild sedation, gastrointestinal upset, and some skin irritation at the site of the thermal heat probe, were observed during the study or were reported by the owners after study completion. All dogs were reported by the owners to show normal behavior by the following day after each treatment. Blood samples were successfully collected from all dogs without any complications. However, a urine sample could not be obtained within the allotted study time from dog 11 after the placebo/tramadol treatment. Average (range) plasma fluconazole concentrations were 4.5 (3.2-6.8) $\mu \mathrm{g} / \mathrm{ml}$ before tramadol administration, 4.9 (3.4-7.0) $\mu \mathrm{g} / \mathrm{ml} 1$ hour after tramadol administration, and $6.0(3.9-9.8) \mu \mathrm{g} / \mathrm{ml} 4$ hours after tramadol administration, indicating good compliance by the owners with fluconazole pretreatment dosing. Urine and plasma samples from one $\operatorname{dog}(\operatorname{dog} 7)$ obtained after fluconazole pretreatment did not show quantifiable tramadol or metabolite concentrations. However, the same
TABLE 2

Inhibitors of M1 and/or M2 formation from tramadol by pooled dog liver microsomes categorized by inhibition potency

Inhibitors are listed according to whether they decrease the indicated activities by at least $50 \%$ (compared with no inhibitor added) at $1 \mu \mathrm{M}$ inhibitor concentration and/or also at $100 \mu \mathrm{M}$ inhibitor concentration. Inhibitors are also grouped by whether they inhibit M1 formation only, $M 2$ formation only, or both $M 1$ and M2 formation by at least $50 \%$ at that inhibitor concentration. The inhibition data used are shown in Table 1.

\begin{tabular}{ccc}
\hline Compounds Inhibiting M1 & Compounds Inhibiting Both & Compounds Inhibiting \\
Formation (Only) by $>50 \%$ & M1 and M2 Formation & M2 Formation (Only) \\
& by $>50 \%$ & by $>50 \%$ \\
\hline
\end{tabular}

Inhibitor concentration:

$1 \mu \mathrm{M}$

Bupivacaine

Citalopram

Imipramine

Ketoconazole

Desipramine

Levomedetomidine

Maropitant

Paroxetine

Quinidine

Trifluoperazine

Inhibitor concentration:

$100 \mu \mathrm{M}$

Acepromazine

Chloramphenicol

Cimetidine

Curcumin

Dopamine

Fentanyl

Firocoxib

Fluoxetine

Lorazepam

Maropitant

Metoclopramide

Omeparazole

Oxazepam

Phenacetin

Quinidine

Terbinafine

Trazadone

Vinblastine

$\begin{array}{ll}\text { Amytriptylline } & \begin{array}{l}\text { Artemisinin } \\ \text { Carprofen }\end{array} \\ \text { Atipamezole } & \text { Dexmedetomidine } \\ \text { Bupivacaine } & \text { Fluconazole } \\ \text { Buprenorphine } & \text { Propofol } \\ \text { Bupropion } & \text { Selegiline } \\ \text { Citalopram } & \\ \text { Clopidrogel } & \\ \text { Chloramphenicol } & \\ \text { Deracoxib } & \\ \text { Desipramine } & \\ \text { Dextromethorphan } & \\ \text { Diazepam } & \\ \text { Doxepin } & \\ \text { Diphenhydramine } & \\ \text { Efavirenz } & \\ \text { Fluconazole } & \\ \text { Fluoxetine } & \\ \text { Imipramine } & \\ \text { Ketamine } & \\ \text { Ketoconazole } & \\ \text { Ketotifen } & \\ \text { Levomedetomidine } & \\ \text { Methadone } & \\ \text { Midazolam } & \\ \text { Paroxetine } & \\ \text { Quinine } & \\ \text { Trifluoperazine } & \\ \text { Xylazine } & \end{array}$

plasma samples contained fluconazole concentrations that were similar to concentrations reported for other dogs. Consequently, it was concluded that this dog had not received the intended dose of tramadol after the fluconazole dose; therefore, the results for this dog were not included in the final analysis.

Effect of Fluconazole on Plasma and Urine Concentrations of Tramadol and Metabolites. Median (25\%-75\%) concentrations and fluconazole/placebo GMR (90\% confidence interval) values for tramadol and metabolites measured in plasma samples 1 and 4 hours after tramadol administration are given in Tables 3 and 4, respectively. Similar summary values for urine samples collected at 4 hours are shown in Table 5. One hour after tramadol administration (Table 3) the median plasma concentration of M1 (but not any other compound measured) showed a small but statistically significant $(P=0.047)$ increase after fluconazole pretreatment compared with placebo pretreatment. However, 4 hours after tramadol administration (Table 4 ), there were marked and statistically significant increases in median plasma tramadol (31fold higher), M1 (39-fold higher), and M1 conjugate (11-fold higher) concentrations. Conversely, there were marked and statistically significant decreases in median plasma M2 (11-fold lower), M2 conjugate 
A

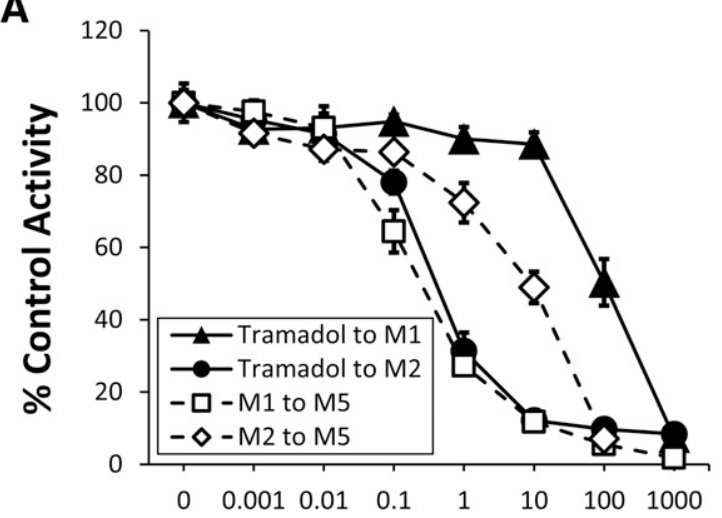

Fluconazole Concentration $(\mu \mathrm{M})$

\section{B}

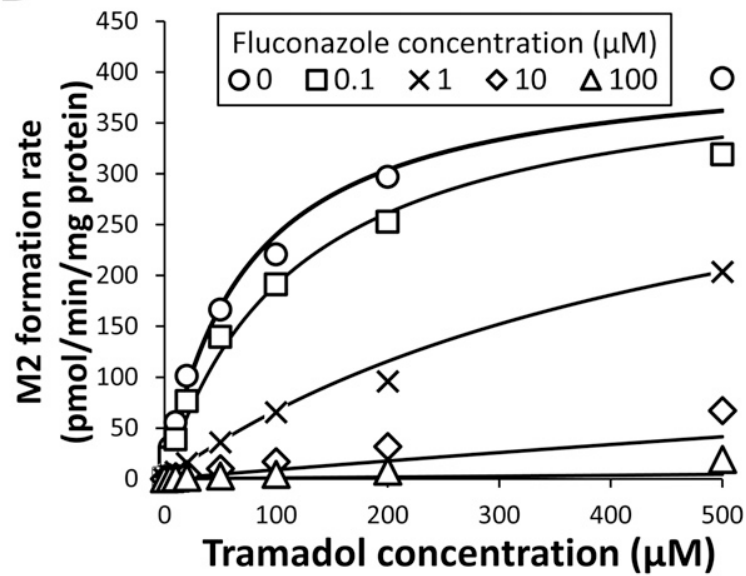

Fig. 1. (A) Selective inhibition of the major primary (tramadol to M1 and tramadol to M2) and secondary (M1 to M5 and M2 to M5) P450-dependent metabolic pathways by fluconazole in pooled DLMs $(N=59)$. Shown are the rates of metabolite formation (mean \pm S.D. of triplicate independent determinations) in the presence of inhibitor (0.001-1000 $\mu \mathrm{M}$ concentration) expressed as a percentage of the formation rate without inhibitor (control activity). (B) Data used to estimate the inhibition constant $\left(K_{\mathrm{i}}\right)$ for inhibition of $\mathrm{M} 2$ formation from tramadol by fluconazole in pooled $(N=59)$ DLMs. Shown are the rates of metabolite formation (mean \pm S.D. of triplicate determinations) in the absence and presence of inhibitor $(0.1,1,10$, and $100 \mu \mathrm{M}$ fluconazole), each at different substrate concentrations $(5,10,20,50$, 100,200 , and $500 \mu \mathrm{M}$ tramadol). The data best fit a competitive inhibition model by nonlinear regression analysis with a $K_{\mathrm{i}}$ value of $0.11 \pm 0.02 \mu \mathrm{M}$.

(4-fold lower), M5 (3-fold lower), and M5 conjugate (7-fold lower) concentrations. There were no changes in tramadol conjugate concentrations.

The changes observed for individual dogs at 4 hours in plasma unconjugated tramadol, M1, M2, and M5 concentrations with fluconazole versus placebo pretreatment are shown in Fig. 2. The direction of change (i.e., increase) in plasma tramadol and M1 concentrations was identical for all dogs (Fig. 2, A and B). Most dogs also showed a decrease in M2 and M5 concentrations (Fig. 2, C and D). However, dog 12 showed an increase in plasma M2 and M5 concentrations, while dog 9 showed an increase in M5 concentrations, with fluconazole versus placebo pretreatment. Compared with other dogs, dog 12 also showed the lowest plasma M2 concentration, while both dogs 9 and 12 showed the lowest plasma M5 concentrations after placebo pretreatment.

Changes in urine tramadol and metabolite concentrations with fluconazole pretreatment (Table 5) were generally similar to the changes observed (as previously described) for the 4-hour plasma concentrations. There were marked and statistically significant increases in median urinary tramadol (35-fold higher), M1 (33-fold higher), and M1 conjugate (5-fold higher) concentrations. Conversely, there were marked and statistically significant decreases in median urinary M2 (28-fold lower) and M5 (12-fold lower) concentrations. There were no changes in median tramadol, M2 conjugate, or M5 conjugate concentrations.

The changes observed for individual dogs in urinary tramadol, M1, M2, and M5 concentrations with fluconazole pretreatment are shown in Fig. 3. Similar to the 4-hour plasma results, the direction of change (i.e., increase) in plasma tramadol and M1 concentrations was identical for all dogs (Fig. 3, A and B). Most dogs also showed a decrease in M2 and M5 concentrations (Fig. 3, C and D), except for $\operatorname{dog} 1$ where small increases (less than 2-fold) in urinary M2 and M5 concentrations were observed. Dog 1 also showed the highest urinary M2 and M5 concentrations after fluconazole pretreatment when compare with results for all other dogs.

The relative proportions of tramadol, M1, M2, and M5 (summed unconjugated plus conjugated for each) expressed as a molar percentage of the total amount of measured tramadol and metabolites in the 4-hour urine samples from individual dogs are shown in Fig. 4. After placebo pretreatment (Fig. 4A), M5 showed the highest median molar proportion (67\%) followed by M2 (26\%), with tramadol (4\%) and M1 (4\%) showing the lowest median molar percentages. However, after fluconazole pretreatment (Fig. 4B), both tramadol (44\%) and M1 (37\%) showed the highest median molar proportion in urine samples, while M5 (12\%) and M2 (1\%) median molar percentages were much lower.

Finally, the relative concentrations of conjugated to unconjugated tramadol, M1, M2, and M5 in urine expressed as a molar ratio are given in Table 6. After placebo pretreatment, M1 showed a high median molar ratio (8.3) and M5 had a much lower median ratio (1.2), while median ratios were very low $(<0.1)$ for both tramadol and M2. After fluconazole pretreatment, there was a significant reduction in the M1 median molar ratio by $66 \%(P=0.002)$, while the M5 median ratio was unchanged $(P=0.002)$. A decrease and an increase in the median ratios for the tramadol and M2 ratios, respectively, were also observed, although the changes were close to the statistical significance threshold $(P=0.027$ and 0.049), reflecting high interindividual variability in the change.

\section{Discussion}

Few published studies have explored the potential for a beneficial pharmacokinetic interaction to enhance the therapeutic properties of a drug in veterinary patients; some examples include Kukanich et al. (2011) and Radwanski et al. (2011). In most instances, known P450 inhibitors (usually based on human in vitro and in vivo studies) are administered orally to decrease first-pass metabolism and increase systemic availability, thereby decreasing the required dose of a second (often costlier) drug that has poor systemic availability by the oral route. Relatively nonspecific potent P450 inhibitors can often achieve this goal. However, in this study the primary goal was to increase plasma concentrations of an active metabolite (M1) that is formed by P450s. Therefore, we needed a more P450-selective approach to inhibit alternative pathways of tramadol and M1 metabolism, without inhibiting M1 formation from tramadol.

Rather than simply choosing P450-selective inhibitors for in vivo testing based solely on available human data, we first used a speciesspecific in vitro approach with canine liver microsomes to identify pathway-selective inhibitor candidates. This was followed by a proofof-principle study in healthy dogs to test the effect of the best candidate on tramadol metabolism in vivo. After screening a large 
TABLE 3

Plasma concentrations (nanograms per milliliter) of tramadol, M1, M2, and M5 and their respective conjugates measured 1 hour after oral administration of tramadol (5 mg/kg b.wt.) to $11 \mathrm{dogs}$ (twice), and after pretreatment with fluconazole and placebo, separated by a 2-week washout period

Conjugate concentrations are given as mass equivalents of the respective parent (unconjugated) compound. The data are presented as the median and the 25\%-75\% interquartile range of

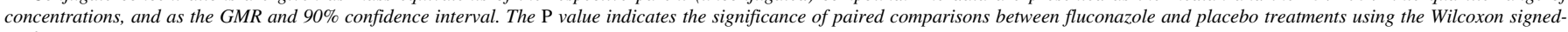
rank test.

\begin{tabular}{|c|c|c|c|c|c|c|c|}
\hline \multirow{3}{*}{ Analyte } & \multicolumn{5}{|c|}{ Plasma Concentration (ng/ml) } & \multirow{2}{*}{\multicolumn{2}{|c|}{$\begin{array}{c}\text { Plasma Concentration Ratio } \\
\text { (Fluconazole/Placebo) }\end{array}$}} \\
\hline & \multicolumn{2}{|c|}{ Placebo } & \multicolumn{2}{|c|}{ Fluconazole } & \multirow{2}{*}{$P$ Value } & & \\
\hline & Median & $25 \%-75 \%$ & Median & $25 \%-75 \%$ & & GMR & $90 \% \mathrm{CI}$ \\
\hline $\mathrm{T}$ & 0.0 & $(0.0-3.8)$ & 3.0 & $(0.0-305)$ & 0.11 & 6.6 & $(1.1-40)$ \\
\hline T conj. & 0.0 & $(0.0-0.0)$ & 0.0 & $(0.0-13)$ & 0.094 & 3.7 & $(1.1-12)$ \\
\hline M1 & 0.0 & $(0.0-0.0)$ & 2.3 & $(0.0-77)$ & $0.047 *$ & 6.1 & $(1.7-21)$ \\
\hline M1 conj. & 2.5 & $(0.0-7.7)$ & 0.0 & $(0.0-191)$ & 0.91 & 1.2 & $(0.25-5.5)$ \\
\hline M2 & 0.0 & $(0.0-47)$ & 0.0 & $(0.0-6.7)$ & 0.31 & 0.51 & $(0.12-2.1)$ \\
\hline M2 conj. & 1.1 & $(0.0-5.8)$ & 0.0 & $(0.0-1.3)$ & 0.15 & 0.41 & $(0.15-1.1)$ \\
\hline M5 & 3.9 & $(0.0-81)$ & 0.0 & $(0.0-8.9)$ & 0.078 & 0.25 & $(0.069-0.92)$ \\
\hline M5 conj. & 7.5 & $(1.6-169)$ & 0.0 & $(0.0-14)$ & 0.13 & 0.12 & $(0.025-0.57)$ \\
\hline
\end{tabular}

$\mathrm{CI}$, confidence interval; conj., conjugate; $\mathrm{T}$, tramadol.

*A $P$ value of less than 0.05 is considered statistically significant.

number of compounds, we were able to identify only one compound (fluconazole) that potently ( $\mathrm{IC}_{50}<1 \mu \mathrm{M}$ ) inhibited the formation of M2 from tramadol and M5 from M1 while minimally affecting the formation of M1 from tramadol $\left(\mathrm{IC}_{50}>100 \mu \mathrm{M}\right)$. There were also several other compounds (artemisinin, carprofen, dexmedetomidine, and propofol) that showed the desired selectivity but only at the higher inhibitor concentration tested $(100 \mu \mathrm{M})$. Although we prioritized fluconazole for further in vitro $\left(\mathrm{IC}_{50}\right.$ and $\left.K_{\mathrm{i}}\right)$ and in vivo testing because of higher inhibition potency, these other compounds could be considered for future evaluation.

The in vivo study pharmacokinetic results completely confirmed the predictions we derived from the in vitro model (summarized in Fig. 5). Markedly higher plasma and urine tramadol concentrations are consistent with overall increased systemic availability of orally administered tramadol via inhibition of metabolism to M2. In prior work (Perez Jimenez et al., 2016), we showed that M2 formation is mediated primarily by CYP2B11 and CYP3A12, which are both highly expressed in tissues that contribute to first-pass extraction of drug after oral administration, including intestinal mucosa and liver (Heikkinen et al., 2015). A previous study showed that the systemic availability of tramadol in dogs after oral administration (at the same dose used here) is very low, averaging only $2.6 \%$ (KuKanich et al., 2017). Oral coadministration of tramadol in that study with P450 inhibitors, including ketoconazole and cimetidine, resulted in about a 5-fold increase in tramadol systemic availability to average $18 \%$ and $20 \%$, respectively. Although we did not measure full plasma concentration-time profiles (i.e., area under the curve) to allow for an accurate calculation of relative systemic availability, plasma tramadol concentrations in this study measured at 1 and 4 hours increased on average by 5.6-fold and 31-fold, respectively, with fluconazole. Consequently, enhancement of systemic availability by fluconazole was at least similar to, or perhaps as much as 5-fold greater than, systemic availability for ketoconazole or cimetidine (i.e., to as high as about $80 \%$ ).

In addition to higher tramadol concentrations, we also observed marked (up to 40-fold) increases in plasma and urine M1 concentrations with fluconazole. This effect is likely a consequence of increased CYP2D15 substrate (i.e., tramadol) availability because of shunting of tramadol metabolism away from the M2 pathway, as well as inhibition of further metabolism of M1 to M5. M1 can also be metabolized by conjugation pathways, including glucuronidation and sulfation (Grond and Sablotzki, 2004). In agreement with prior studies (Grond and Sablotzki, 2004; Giorgi et al., 2009), our results showed that M1 is primarily found as conjugates in the urine (Tables 5 and 6), extending those findings to include plasma conjugates (Tables 3 and 4). Consequently, conjugation is likely an important mechanism for elimination of M1 in dogs, and inhibition of M1 conjugation by fluconazole would be another mechanism to explain increased M1 plasma and urine concentrations. Although we did not directly test whether fluconazole inhibited M1 conjugation, we did observe a $66 \%$ decrease in the M1 conjugated/unconjugated concentration ratio with fluconazole treatment compared with placebo, which is consistent with enzymatic inhibition. However, these lower ratios could also be explained by saturation of conjugation pathways resulting from increased substrate (i.e., M1) formation. Interestingly, M1 was shown previously to be glucuronidated primarily by UGT2B7 in human liver (Lehtonen et al., 2010), and fluconazole is a selective inhibitor of UGT2B7 (Uchaipichat et al., 2006). M1 is also conjugated by sulfation, although this appears to occur primarily in the intestinal mucosa rather than in the liver or kidney, and thus may not contribute greatly to systemic clearance, at least in humans (Rasool et al., 2017). It is unclear whether fluconazole can inhibit sulfotransferases.

Several other research studies have attempted to modify tramadol and metabolite pharmacokinetics in dogs by coadministering selected food extracts or drugs (Giorgi et al., 2011; KuKanich et al., 2017). In the first study (Giorgi et al., 2011), tramadol was coadministered with dried grapefruit juice; the premise was that certain grapefruit constituents known to inhibit human CYP3A would also inhibit canine CYP3A, thereby promoting metabolism of tramadol to M1 by CYP2D. However, the study appreciated only a negligible effect of grapefruit on tramadol and M1 concentrations and no effect on M2 or M5 concentrations (Giorgi et al., 2011). Although the authors of that study had no direct evidence at the time, their underlying assumptions were partially correct in that canine CYP2D15 metabolizes tramadol to M1 and canine CYP3A12 contributes to the metabolism of tramadol to M2 (Perez Jimenez et al., 2016). However, additional canine P450s, particularly CYP2B11, metabolize tramadol to M2, which may have limited the impact of any CYP3A inhibition by grapefruit. Interestingly, administration of bergamottin, the principal $\mathrm{P} 450$ inhibitor in grapefruit juice, to beagle dogs for 10 days resulted in a modest decrease in hepatic microsomal CYP3A activity, consistent with enzyme inhibition (Sahi et al., 2002). However, there was also a 
TABLE 4

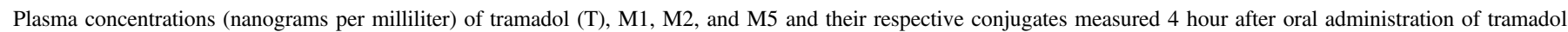
( $5 \mathrm{mg} / \mathrm{kg}$ b.wt.) to $11 \mathrm{dogs}$ (twice), and after pretreatment with fluconazole and placebo, separated by a 2-week washout period

Conjugate concentrations are given as mass equivalents of the respective parent (unconjugated) compound. The data are presented as the median and the $25 \%-75 \%$ interquartile range of

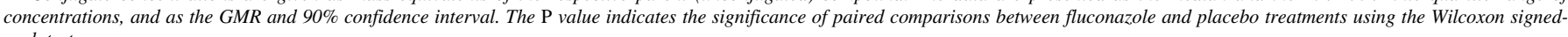
rank test.

\begin{tabular}{|c|c|c|c|c|c|c|c|}
\hline \multirow{3}{*}{ Analyte } & \multicolumn{5}{|c|}{ Plasma Concentration (ng/ml) } & \multirow{2}{*}{\multicolumn{2}{|c|}{$\begin{array}{c}\text { Plasma Concentration Ratio } \\
\text { (Fluconazole/Placebo) }\end{array}$}} \\
\hline & \multicolumn{2}{|c|}{ Placebo } & \multicolumn{2}{|c|}{ Fluconazole } & \multirow{2}{*}{$P$ Value } & & \\
\hline & Median & $25 \%-75 \%$ & Median & $25 \%-75 \%$ & & GMR & $90 \% \mathrm{CI}$ \\
\hline $\mathrm{T}$ & 6.4 & $(3.0-20)$ & 215 & $(59-502)$ & $<0.001^{*}$ & 32 & $(11-98)$ \\
\hline $\mathrm{T}$ conj. & 0.0 & $(0.0-5.5)$ & 13 & $(0.0-57)$ & 0.13 & 5.8 & $(0.79-42)$ \\
\hline M1 & 0.0 & $(0.0-2.8)$ & 54 & $(38-68)$ & $<0.001^{*}$ & 40 & $(21-76)$ \\
\hline M1 conj. & 34 & $(13-67)$ & 325 & $(121-764)$ & $<0.001^{*}$ & 12 & $(5.8-24)$ \\
\hline M2 & 83 & $(33-215)$ & 5.4 & $(1.1-18)$ & $0.002 *$ & 0.083 & $(0.033-0.21)$ \\
\hline M2 conj. & 8.8 & $(0.0-40.9)$ & 2.2 & $(0.0-3.8)$ & $0.049 *$ & 0.22 & $(0.061-0.82)$ \\
\hline M5 & 79 & $(40-88)$ & 10 & $(5.7-12)$ & $0.005^{*}$ & 0.27 & $(0.094-0.79)$ \\
\hline M5 conj. & 825 & (497-1195) & 54 & $(21-109)$ & $0.002 *$ & 0.12 & $(0.042-0.32)$ \\
\hline
\end{tabular}

CI, confidence interval; conj., conjugate; $\mathrm{T}$, tramadol.

*A $P$ value of less than 0.05 was considered statistically significant.

substantial increase in hepatic CYP2B activities, presumably caused by enzyme induction.

In the second study, tramadol was orally coadministered with ketoconazole or cimetidine (both assumed to be CYP3A inhibitors) to evaluate effects on tramadol and metabolite pharmacokinetics (KuKanich et al., 2017). The authors in that study observed increases in tramadol plasma concentrations with both inhibitors, but still well below the minimum tramadol concentration of $228 \mathrm{ng} / \mathrm{ml}$ associated with analgesia in humans (Grond et al., 1999). There were also some increases in mean maximal plasma concentrations $\left(C_{\max }\right)$ for M1 from 1.8 to $2.5 \mathrm{ng} / \mathrm{ml}$ (with ketoconazole) and to $6.6 \mathrm{ng} / \mathrm{ml}$ (with cimetidine). However, those M1 concentrations were below what was considered the minimal effective concentration $(10 \mathrm{ng} / \mathrm{ml}$ ) in humans (Scott and Perry, 2000). Ketoconazole also resulted in significant increases in plasma $\mathrm{M} 2$ concentrations ( $C_{\max }$ and area under the curve) and decreases in M5 concentrations ( $C_{\max }$ but not area under the curve), which contrasts with the decreases in both M2 and M5 plasma concentrations observed in this study. We have previously shown that M2 is converted to M5 exclusively by CYP2D15 (Perez Jimenez et al., 2018). In contrast to fluconazole, which had a minimal effect on M2 metabolism to M5 at concentrations up to $10 \mu \mathrm{M}$ in this study (Fig. 1A), ketoconazole was reported to inhibit CYP2D15 with a potency $\left(\mathrm{IC}_{50}=0.47 \mu \mathrm{M}\right)$ that was similar to or greater than potencies for all other P450s evaluated, including CYP1A, CYP2C, CYP2B, and CYP3A (Aidasani et al., 2008). Consequently, increased plasma M2 concentrations observed with coadministration of ketoconazole with tramadol could be explained by inhibition of CYP2D15-mediated metabolism of M2 to M5.

The median plasma M1 concentration 4 hours after tramadol with fluconazole pretreatment was $54 \mathrm{ng} / \mathrm{ml}$ in the current study, which is higher than that achieved in all of the previous studies evaluating effects of tramadol on nociceptive thresholds (Kukanich and Papich, 2011; Kögel et al., 2014; Schütter et al., 2017). This is also within the plasma M1 concentration range of $36-84 \mathrm{ng} / \mathrm{ml}$ associated with clinical pain relief in humans (Lehmann et al., 1990; Grond et al., 1999); however, it is somewhat less than the median effective plasma M1 concentration of $113 \mathrm{ng} / \mathrm{ml}$ that was reported for cats using a thermal threshold testing method (Pypendop et al., 2009).

There are several limitations to this study. First, we measured tramadol concentrations at only two time points, and thus it cannot

TABLE 5

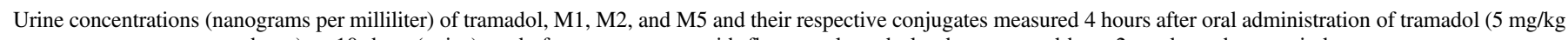
b.wt.) to $10 \mathrm{dogs}$ (twice), and after pretreatment with fluconazole and placebo separated by a 2-week washout period

Conjugate concentrations are given as mass equivalents of the respective parent (unconjugated) compound. The data are presented as the median and the $25 \%-75 \%$ interquartile range of

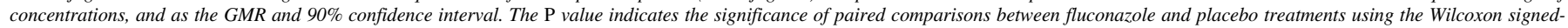
rank test.

\begin{tabular}{|c|c|c|c|c|c|c|c|}
\hline \multirow{3}{*}{ Analyte } & \multicolumn{5}{|c|}{ Urine Concentration (ng/ml) } & \multirow{2}{*}{\multicolumn{2}{|c|}{$\begin{array}{c}\text { Urine Concentration Ratio } \\
\text { (Fluconazole/Placebo) }\end{array}$}} \\
\hline & \multicolumn{2}{|c|}{ Placebo } & \multicolumn{2}{|c|}{ Fluconazole } & \multirow{2}{*}{$P$ Value } & & \\
\hline & Median & $25 \%-75 \%$ & Median & $25 \%-75 \%$ & & GMR & $90 \% \mathrm{CI}$ \\
\hline $\mathrm{T}$ & 144 & $(11-498)$ & 1528 & $(881-8856)$ & $0.002 *$ & 36 & $(10-127)$ \\
\hline $\mathrm{T}$ conj. & 0.0 & $(0.0-71)$ & 0.0 & $(0.0-8.2)$ & 0.31 & 0.87 & $(0.19-3.9)$ \\
\hline M1 & 34 & $(11-102)$ & 728 & $(328-2135)$ & $0.002 *$ & 34 & $(12-94)$ \\
\hline M1 conj. & 473 & $(126-1330)$ & 2048 & $(895-6013)$ & $0.01 *$ & 6.4 & $(2.2-18.9)$ \\
\hline M2 & 1284 & $(522-3570)$ & 59 & $(8.2-437)$ & $0.01 *$ & 0.034 & $(0.011-0.11)$ \\
\hline M2 conj. & 218 & $(0.0-717)$ & 15 & $(6.3-39)$ & 0.13 & 0.40 & $(0.055-2.9)$ \\
\hline M5 & 2968 & $(1872-3802)$ & 224 & $(81-758)$ & $0.006^{*}$ & 0.076 & $(0.026-0.22)$ \\
\hline M5 conj. & 4227 & $(859-10,186)$ & 372 & $(168-3299)$ & 0.32 & 0.28 & $(0.056-1.4)$ \\
\hline
\end{tabular}

CI, confidence interval; conj., conjugate; $\mathrm{T}$, tramadol.

*A $P$ value of less than 0.05 was considered statistically significant. 
A
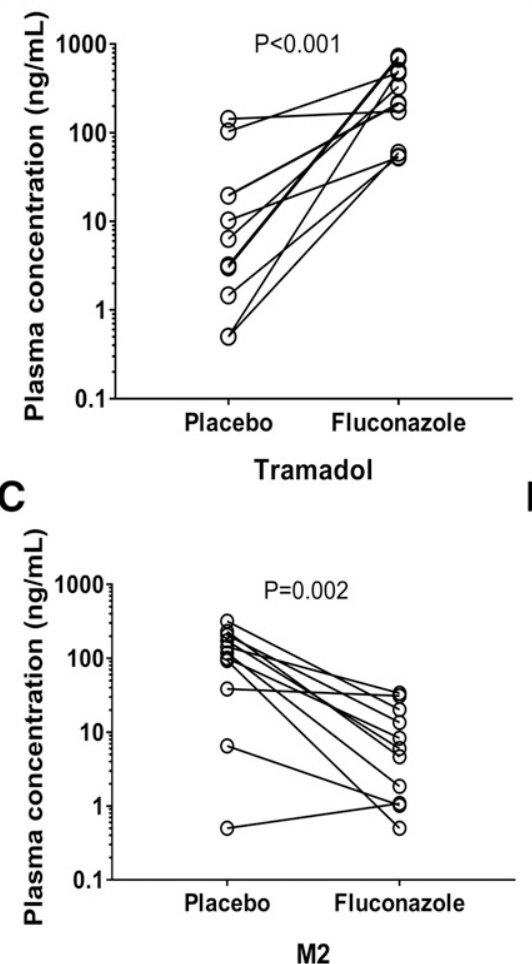

B
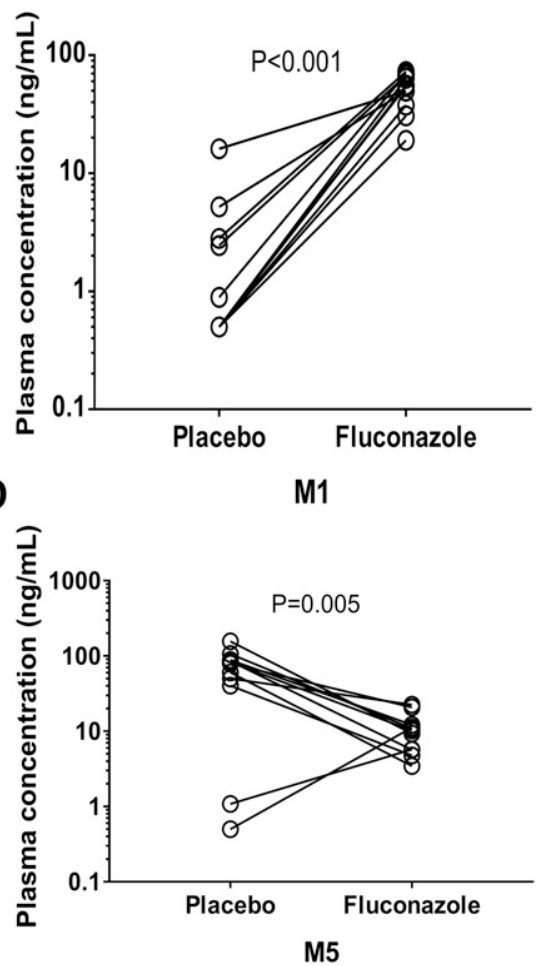

Fig. 2. Plasma concentrations (nanograms per milliliter) of tramadol (A), M1 (B), M2 (C), and M5 (D) measured 4 hours after oral administration of tramadol $(5 \mathrm{mg} / \mathrm{kg}$ b.wt.) to $11 \mathrm{dogs}$ (twice), and after pretreatment with fluconazole and placebo, separated by a 2-week washout period. Open circles are plasma concentration values for each dog measured after placebo and fluconazole pretreatments, connected by a line to show the change that occurred within individual subjects. Also shown are $P$ values obtained using the Wilcoxon signed-rank test comparing fluconazole to placebo pretreatments. be determined whether tramadol half-life is more prolonged with fluconazole coadministration. This would dictate whether dose and/or dose interval adjustments are needed with repeated dosing, which is more commonly used for treatment of pain. Second, we only evaluated a single tramadol dose, and there is some prior evidence for increased tramadol clearance in dogs with repeated
A

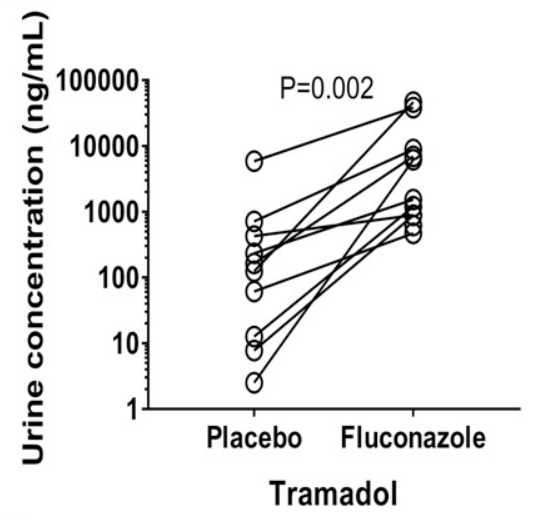

C

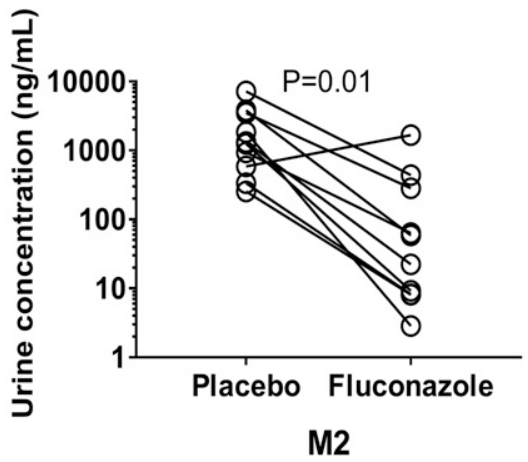

B
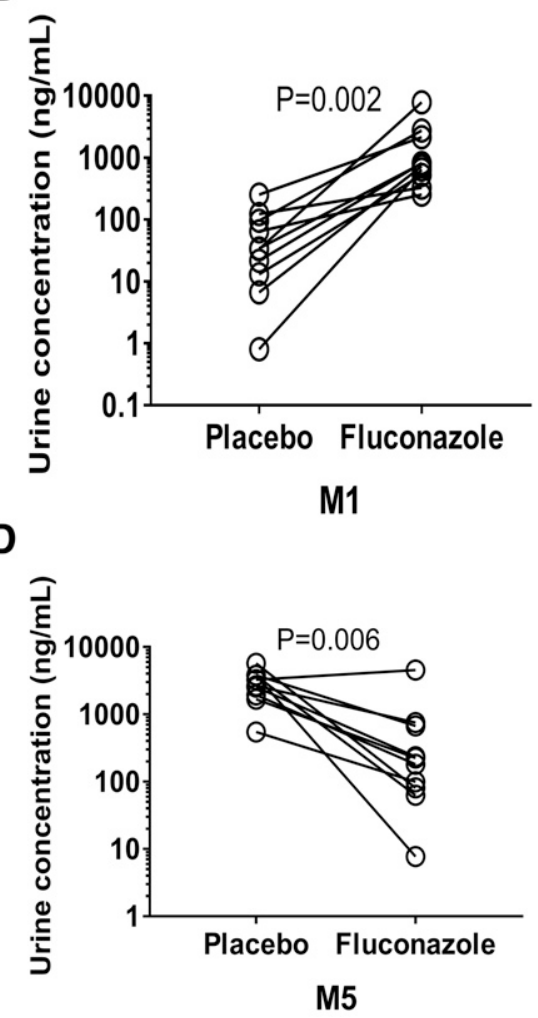

Fig. 3. Urine concentrations (nanograms per milliliter) of tramadol (A), M1 (B), M2 (C), and M5 (D) measured 4 hours after oral administration of tramadol $(5 \mathrm{mg} / \mathrm{kg}$ b.wt. $)$ to $10 \mathrm{dogs}$ (twice), and after pretreatment with fluconazole and placebo, separated by a 2 -week washout period. Note that a urine sample could not be obtained from dog 11 after placebo pretreatment. Open circles are urine concentration values for each dog measured after placebo and fluconazole pretreatments, connected by a line to show the change that occurred within individual subjects. Also shown are $P$ values obtained using the Wilcoxon signed-rank test comparing fluconazole to placebo pretreatments. 
A

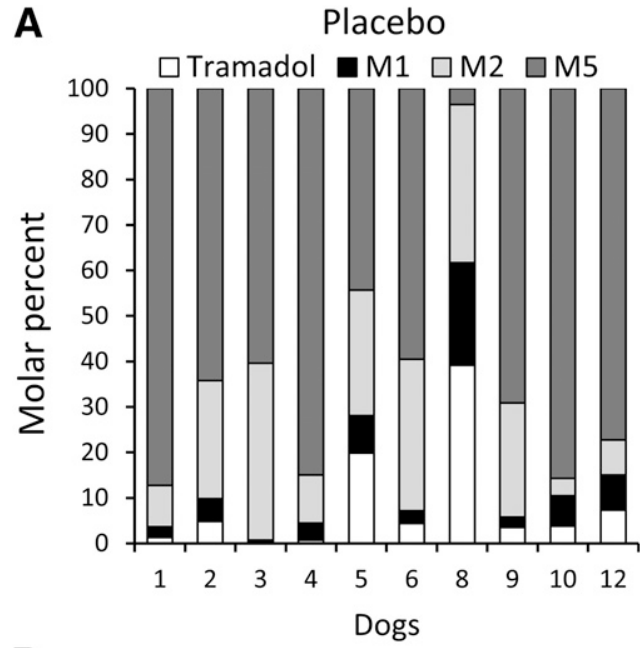

B

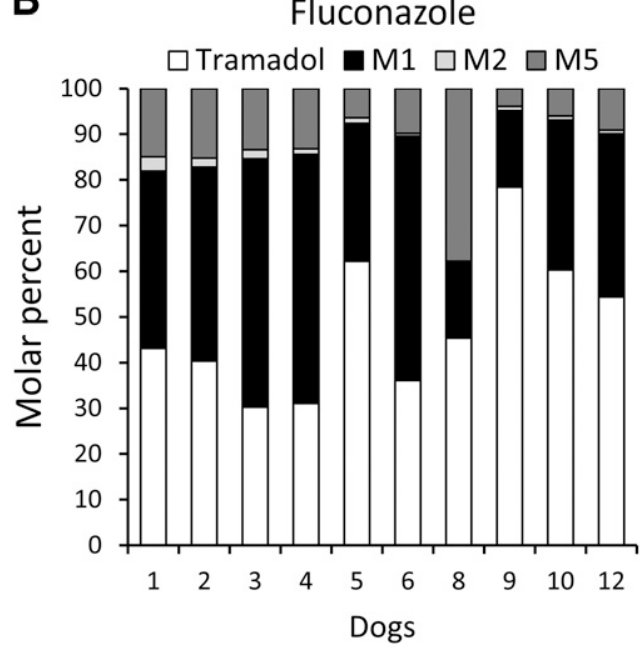

Fig. 4. Relative proportions of tramadol, M1, M2, and M5 (unconjugated plus conjugated) measured in urine samples 4 hours after oral administration of tramadol ( $5 \mathrm{mg} / \mathrm{kg} \mathrm{b}$.wt.) to $10 \mathrm{dogs}$ (twice), and after pretreatment with placebo (A) and fluconazole (B), separated by a 2-week washout period. Each stacked bar show values for individual dogs expressed as a molar percentage of total measured compounds for that urine sample.

dosing (Matthiesen et al., 1998). Third, it is not yet clear whether increased tramadol and M1 concentrations will lead to increased analgesic efficacy. Consequently, future studies are needed to

TABLE 6

Urine conjugated/unconjugated molar ratios of tramadol, M1, M2, and M5 measured 4 hours after administration of tramadol $(5 \mathrm{mg} / \mathrm{kg} \mathrm{b}$.wt.) orally to $10 \mathrm{dogs}$ (twice), and after pretreatment with fluconazole and placebo, separated by a 2-week washout period

The data are presented as the median and the 25\%-75\% interquartile range of concentrations. The $\mathrm{P}$ value indicates the significance of paired comparisons between fluconazole and placebo treatments using the Wilcoxon signed-rank test.

\begin{tabular}{|c|c|c|c|c|c|}
\hline \multirow{3}{*}{ Analyte } & \multicolumn{5}{|c|}{ Urine Conjugated/Unconjugated Molar Ratio } \\
\hline & \multicolumn{2}{|c|}{ Placebo } & \multicolumn{2}{|c|}{ Fluconazole } & \multirow[b]{2}{*}{$P$ Value } \\
\hline & Median & $25 \%-75 \%$ & Median & $25 \%-75 \%$ & \\
\hline Tramadol & 0.014 & $(0.008-0.57)$ & 0.002 & $(0.0002-0.022)$ & $0.027 *$ \\
\hline M1 & 8.3 & $(4.6-16)$ & 2.8 & $(1.1-3.9)$ & $0.002 *$ \\
\hline M2 & 0.08 & $(0.01-0.34)$ & 0.44 & $(0.20-1.08)$ & $0.049 *$ \\
\hline M5 & 1.2 & $(0.5-3.6)$ & 1.7 & $(0.7-3.6)$ & 0.43 \\
\hline
\end{tabular}

*A $P$-value of less than 0.05 was considered statistically significant.
Placebo

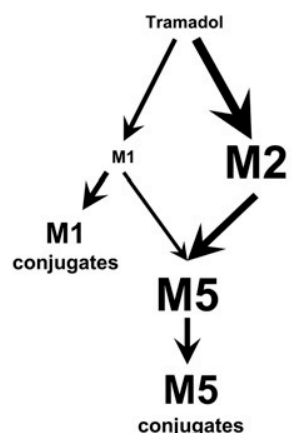

Fluconazole

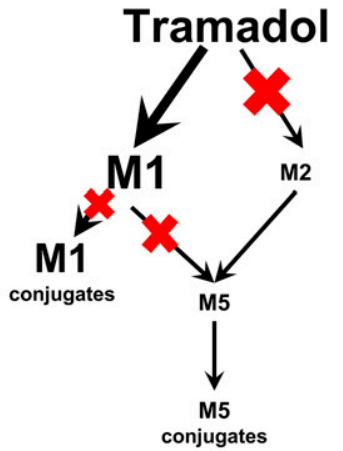

Fig. 5. Effect of fluconazole on tramadol metabolism in dogs based on the results of this study. The primary pathway for tramadol metabolism is $N$-demethylation to M2 followed by $O$-demethylation to M5. M2 and M5 (and their phase 2 metabolism conjugates) are the most abundant metabolites found in dog plasma and urine. $O$ demethylation of tramadol to M1 followed by $N$-demethylation to M5 is a minor pathway, and only low concentrations of M1 and its conjugates are detected in plasma and urine. Fluconazole was identified as a potent inhibitor of M2 formation from tramadol and M5 formation from M1 in DLMs without inhibiting the other two pathways. Oral pretreatment of dogs with fluconazole markedly increased plasma and urine concentrations of tramadol and M1 and decreased M2 and M5 concentrations.

determine whether adding fluconazole can enhance the analgesic efficacy of tramadol in healthy dogs (such as through nociceptive threshold testing) and clinical patients experiencing pain.

\section{Authorship Contributions}

Participated in research design: Perez Jimenez, Mealey, Grubb, Greene, Court.

Conducted experiments: Perez Jimenez, Kukanich, Joo.

Performed data analysis: Perez Jimenez, Court.

Wrote or contributed to the writing of the manuscript: Perez Jimenez, Mealey, Grubb, Greene, Court, Kukanich, Joo.

\section{References}

Aidasani D, Zaya MJ, Malpas PB, and Locuson CW (2008) In vitro drug-drug interaction screens for canine veterinary medicines: evaluation of cytochrome P450 reversible inhibition. Drug Metab Dispos 36:1512-1518

Aronson MD (1997) Nonsteroidal anti-inflammatory drugs, traditional opioids, and tramadol: contrasting therapies for the treatment of chronic pain. Clin Ther 19:420-432, discussion 367368 .

Cagnardi P, Villa R, Zonca A, Gallo M, Beccaglia M, Luvoni GC, Vettorato E, Carli S, Fonda D, and Ravasio G (2011) Pharmacokinetics, intraoperative effect and postoperative analgesia of tramadol in cats. Res Vet Sci 90:503-509.

Court MH, Von Moltke LL, Shader RI, and Greenblatt DJ (1997) Biotransformation of chlor zoxazone by hepatic microsomes from humans and ten other mammalian species. Biopharm Drug Dispos 18:213-226.

Davila D, Keeshen TP, Evans RB, and Conzemius MG (2013) Comparison of the analgesic efficacy of perioperative firocoxib and tramadol administration in dogs undergoing tibial plateau leveling osteotomy. J Am Vet Med Assoc 243:225-231.

Delgado C, Bentley E, Hetzel S, and Smith LJ (2014) Comparison of carprofen and tramadol for postoperative analgesia in dogs undergoing enucleation. J Am Vet Med Assoc 245:1375-1381. Evangelista MC, Silva RA, Cardozo LB, Kahvegian MA, Rossetto TC, Matera JM, and Fantoni DT (2014) Comparison of preoperative tramadol and pethidine on postoperative pain in cats undergoing ovariohysterectomy. BMC Vet Res 10:252.

Gaynor JS (2008) Control of cancer pain in veterinary patients. Vet Clin North Am Small Anim Pract 38:1429-1448.

Giorgi M, Del Carlo S, Saccomanni G, Łebkowska-Wieruszewska B, and Kowalski CJ (2009) Pharmacokinetic and urine profile of tramadol and its major metabolites following oral immediate release capsules administration in dogs. Vet Res Commun 33:875-885.

Giorgi M, MacCheroni M, Del Carlo S, Yun HI, and Saccomanni G (2011) Effect of oral co-administration of frozen-dried grapefruit juice on pharmacokinetics of tramadol in dogs. IJVR 12:8-15.

Grond S, Meuser T, Uragg H, Stahlberg HJ, and Lehmann KA (1999) Serum concentrations of tramadol enantiomers during patient-controlled analgesia. Br J Clin Pharmacol 48:254-257.

Grond S and Sablotzki A (2004) Clinical pharmacology of tramadol. Clin Pharmacokinet 43: 879-923.

Heikkinen AT, Friedlein A, Matondo M, Hatley OJ, Petsalo A, Juvonen R, Galetin A, RostamiHodjegan A, Aebersold R, Lamerz J, et al. (2015) Quantitative ADME proteomics-CYP and UGT enzymes in the beagle dog liver and intestine. Pharm Res 32:74-90. 
Kögel B, Terlinden R, and Schneider J (2014) Characterisation of tramadol, morphine and tapentadol in an acute pain model in beagle dogs. Vet Anaesth Analg 41 297-304.

KuKanich B, KuKanich K, and Black J (2017) The effects of ketoconazole and cimetidine on the pharmacokinetics of oral tramadol in greyhound dogs [published correction appears in $J$ Vet Pharmacol Ther (2018) 41:354]. J Vet Pharmacol Ther 40:e54-e61.

Kukanich B, Kukanich KS, and Rodriguez JR (2011) The effects of concurrent administration of cytochrome P-450 inhibitors on the pharmacokinetics of oral methadone in healthy dogs. Vet Anaesth Analg 38:224-230.

Kukanich B and Papich MG (2011) Pharmacokinetics and antinociceptive effects of oral tramadol hydrochloride administration in Greyhounds. Am J Vet Res 72:256-262.

Lamont LA (2008) Multimodal pain management in veterinary medicine: the physiologic basis of pharmacologic therapies. Vet Clin North Am Small Anim Pract 38:1173-1186.

Lehmann KA, Kratzenberg U, Schroeder-Bark B, and Horrichs-Haermeyer G (1990) Postoperative patient-controlled analgesia with tramadol: analgesic efficacy and minimum effective concentrations. Clin J Pain 6:212-220.

Lehtonen P, Sten T, Aitio O, Kurkela M, Vuorensola K, Finel M, and Kostiainen R (2010) Glucuronidation of racemic $O$-desmethyltramadol, the active metabolite of tramadol. Eur $J$ Pharm Sci 41:523-530.

Matthiesen T, Wöhrmann T, Coogan TP, and Uragg H (1998) The experimental toxicology of tramadol: an overview. Toxicol Lett 95:63-71.

Perez Jimenez TE, Mealey KL, Grubb TL, Greene SA, and Court MH (2016) Tramadol metabolism to $O$-desmethyl tramadol (M1) and $N$-desmethyl tramadol (M2) by dog liver microsomes: species comparison and identification of responsible canine cytochrome P-450s (CYPs). Drug Metab Dispos 44:1963-1972.

Perez Jimenez TE, Mealey KL, Schnider D, Grubb TL, Greene SA, and Court MH (2018) Identification of canine cytochrome P-450s (CYPs) metabolizing the tramadol (+)-M1 and (+)-M2 metabolites to the tramadol (+)-M5 metabolite in dog liver microsomes. Pharmacol Ther 41 815-824.

Pypendop BH, Siao KT, and Ilkiw JE (2009) Effects of tramadol hydrochloride on the thermal threshold in cats. Am J Vet Res 70:1465-1470.
Radwanski NE, Cerundolo R, Shofer FS, Hanley MJ, and Court MH (2011) Effects of powdered whole grapefruit and metoclopramide on the pharmacokinetics of cyclosporine in dogs. Am J Vet Res 72:687-693.

Raffa RB, Friderichs E, Reimann W, Shank RP, Codd EE, and Vaught JL (1992) Opioid and nonopioid components independently contribute to the mechanism of action of tramadol, an 'atypical' opioid analgesic. J Pharmacol Exp Ther 260:275-285.

Rasool MI, Bairam AF, Kurogi K, and Liu MC (2017) On the sulfation of $O$-desmethyltramadol by human cytosolic sulfotransferases. Pharmacol Rep 69:953-958.

Sahi J, Reyner EL, Bauman JN, Gueneva-Boucheva K, Burleigh JE, and Thomas VH (2002) The effect of bergamottin on diazepam plasma levels and P450 enzymes in beagle dogs. Drug Metab Dispos 30:135-140.

Schütter AF, Tünsmeyer J, and Kästner SBR (2017) Influence of tramadol on acute thermal and mechanical cutaneous nociception in dogs. Vet Anaesth Analg 44:309-316.

Scott LJ and Perry CM (2000) Tramadol: a review of its use in perioperative pain. Drugs 60 $139-176$.

Subrahmanyam V, Renwick AB, Walters DG, Young PJ, Price RJ, Tonelli AP, and Lake BG (2001) Identification of cytochrome P-450 isoforms responsible for cis-tramadol metabolism in human liver microsomes. Drug Metab Dispos 29:1146-1155.

Uchaipichat V, Winner LK, Mackenzie PI, Elliot DJ, Williams JA, and Miners JO (2006) Quantitative prediction of in vivo inhibitory interactions involving glucuronidated drugs from in vitro data: the effect of fluconazole on zidovudine glucuronidation. Br J Clin Pharmacol 61:427-439. Venkatakrishnan K, Von Moltke LL, and Greenblatt DJ (2001) Human drug metabolism and the cytochromes P450: application and relevance of in vitro models. J Clin Pharmacol 41:1149-1179.

Address correspondence to: Dr. Tania E. Perez Jimenez, Program in Individualized Medicine, Pharmacogenomics Laboratory, Department of Veterinary Clinical Sciences, Washington State University College of Veterinary Medicine, 100 Grimes Way, Pullman, WA 99164. E-mail: teperezjimenez@wsu.edu 